\title{
Characterization of Creep Damage in Metals Using Small Angle Neutron Scattering
}

\author{
E. R. Fuller, Jr., R. J. Fields, T.-J. Chuang, and S. Singhal \\ National Bureau of Standards, Washington, DC 20234
}

Accepted: December 1, 1983

\begin{abstract}
Creep damage in polycrystalline metallic materials can be attributed to cavitation and cracking along the grain interfaces. Theories of creep cavitation that have been developed in recent years are reviewed. Further evaluation and/or refinement of these theories has been retarded by a lack of an experimental counterpart. Small angle neutron scattering studies (SANS) provide one experimental tool which is complementary to others. SANS done at NBS and elsewhere have shown that this technique is suitable for studying nucleation and early stage of growth of creep cavities. This would provide the impetus to further progress in this area.
\end{abstract}

Key words: creep cavitation; creep crack growth; creep damage; creep fracture; high temperature failure of metals; small angle neutron scattering.

\section{Introduction}

At high temperatures and low stresses, metals often fail with an elongation of only a few percent and only a small reduction in area [1] $]^{1}$. This phenomenon occurs even in normally ductile materials like copper and nickel [2]. When metals which have fractured under the above conditions are examined microscopically, it is found that they have cavities and cracks distributed throughout the specimen along grain boundaries as shown in figure 1 . The failure mechanism associated with these cavities is therefore referred to as intergranular creep fracture. It is the dominant

About the Authors, Paper: All four authors are members of organization units within NBS' Center for Materials Science. E. R. Fuller, Jr. and T.-J. Chuang are with the Inorganic Materials Division, R. J. Fields is with the Fracture and Deformation Division, and S. Singhal is a member of the Center's Reactor Radiation Division. The work on which they report was supported by the NBS Office of Nondestructive Evaluation.

\footnotetext{
${ }^{1}$ Numbers in brackets refer to references at the end of this paper.
}

mechanism of long-term fracture of both 304 stainless steel and 2-1/4 $\mathrm{Cr}-1$ Mo steel, as shown in figure 2 [1,3-7], and of many other commercial alloys.

The cavities usually contain inclusions or second phase particles, apparently having nucleated on them. The cavities, once nucleated, grow by the diffusive movement of atoms from the cavity onto and along the grain boundary [8] as shown schematically in figure 3 . This mechanism is equivalent to the stressdirected condensation of vacancies on the cavity. The migration of atoms (or of vacancies) results in the extension of the specimen-allowing the applied force to do work. Most of this work is dissipated by the diffusive fluxes associated with the redistribution of matter [9]; some, however, is stored as the energy associated with the newly created surface area of the cavities. If the load were removed, the specimen would contract as the cavities shrink by sintering. However, it is the nucleation and growth of these cavities that is of interest here as more and more of the grain boundaries are consumed and fracture eventually occurs.

As will be shown, theoretical progress in modeling creep cavitation has greatly outstripped the experimental advances or measurements in this field. At present, there are several viable models that can predict the 

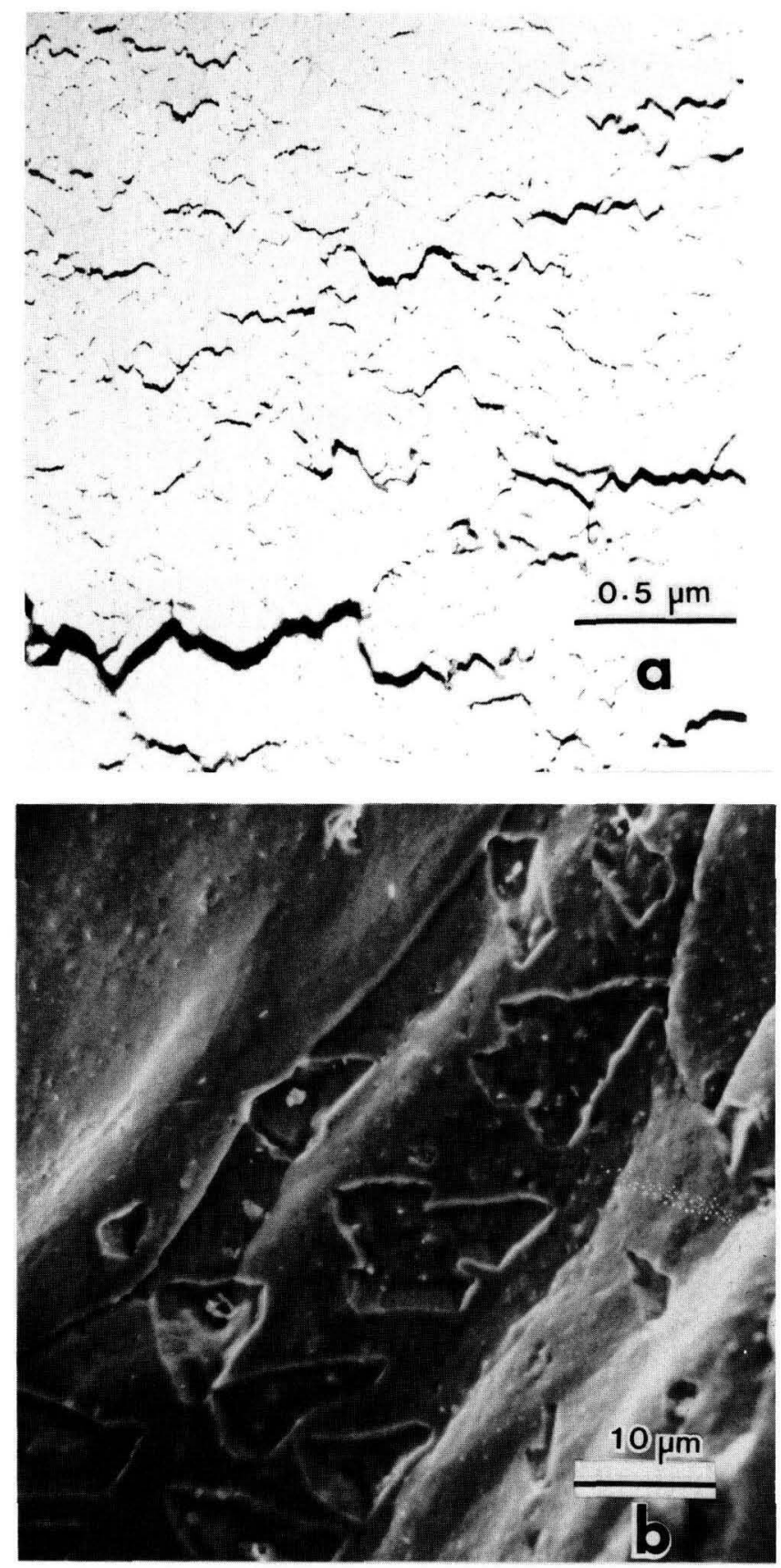

Figure 1-(a) Intergranular cavitation in copper tested at $600^{\circ} \mathrm{C}$ viewed edge-on in an optical microscope. (b) Intergranular cavitation in iron tested at $600{ }^{\circ} \mathrm{C}$, viewed in a scanning electron microscope on a grain boundary which is exposed by a low temperature fracture.

accumulation of damage and the time-to-fracture. Experimental measurements clearly supporting one model or, at least, rationalizing the differences between models, have not been available. Indeed, most experiments have focused on the stress dependence of the time-to-fracture (an integrated result), and have
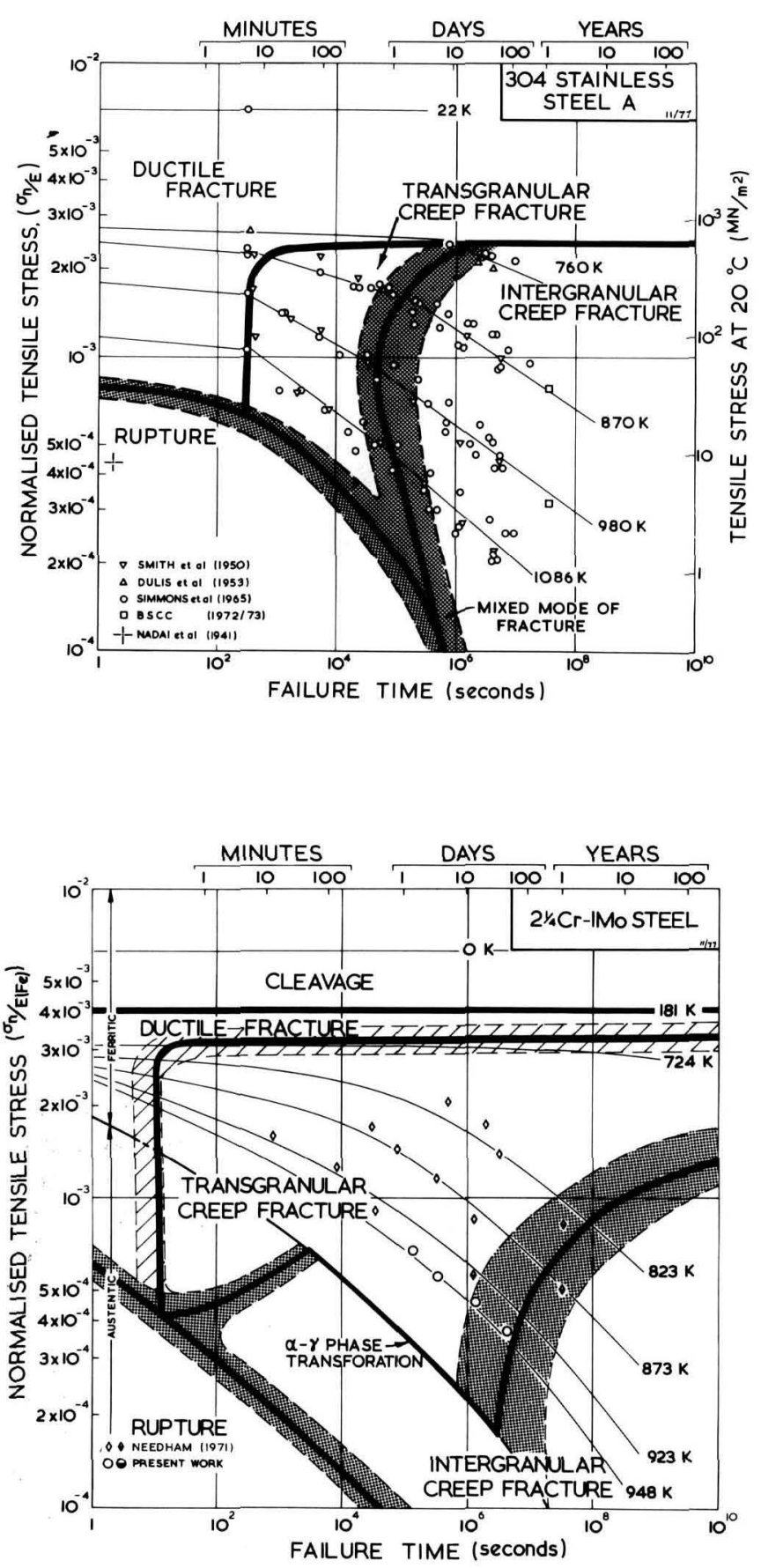

Figure 2-Fracture mechanism maps for 304 stainless steel and $2 \frac{1}{4} \mathrm{Cr}$ 1 Mo steel showing the regions of stress and temperature in which intergranular cavitation occurs.

given little attention to size distributions of cavities and their time dependence. Furthermore, this lack of experimental measurements for small cavity size distributions has hindered refinement of the various theoretical models. 


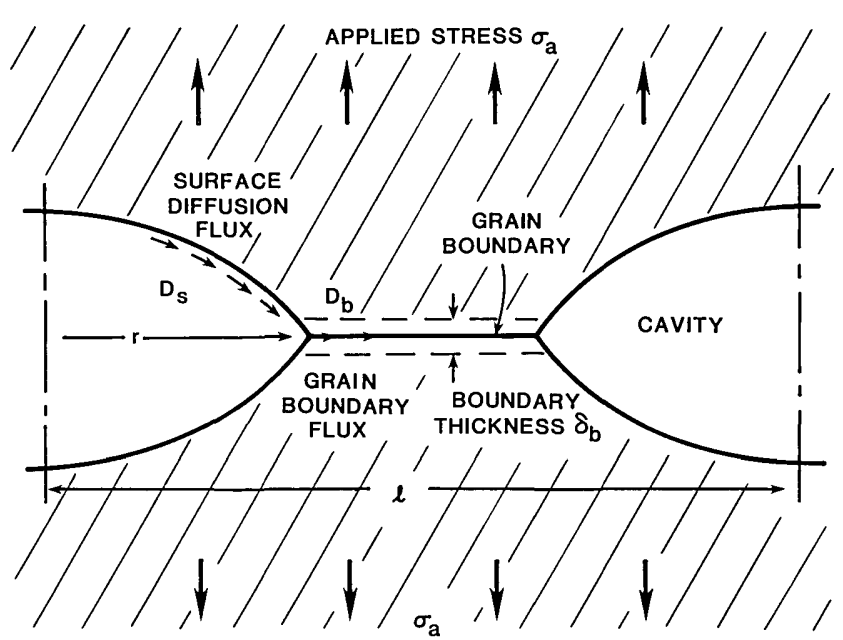

Figure 3-Schematic illustration of the stress-directed flow of material from the cavity surface onto the grain boundary.

In this paper, we present small angle neutron scattering (SANS) results which show that SANS is quantitatively sensitive to these small cavities. These results indicate that SANS may be the new technique to answer many of the pressing questions arising from existing theoretical treatments of creep cavitation. To understand what these questions are and how they have arisen, we first give a brief review of the theoretical developments in this area of fracture.

Historically, developments in creep cavitation have focused primarily on the stress dependence of the time-to-fracture, which, unfortunately, is not directly related to the cavity size information obtained by SANS. To indicate this connection, we demonstrate for several of these models how cavity size varies with time under stress. Following this review, we briefly discuss several experimental techniques for obtaining information on creep cavitation damage and conclude with a section describing our SANS measurements for a 304 type stainless steel.

\section{Modeling of Creep Cavitation}

On the basis of the few available experimental observations, Hull and Rimmer [10] developed a basic model for creep cavitation. They assumed that the cavities are spherical and that diffusion of matter away from them is achieved predominantly by grain boundary diffusion. According to Fick's first law, the flux, $J$, of matter from the cavity to the grain boundary is

$$
J=-\left(D_{\mathrm{b}} / \mathrm{kT} \Omega\right) \nabla \mu
$$

where $D_{\mathrm{b}}$ is the grain boundary diffusivity, $\Omega$ is the atomic volume, and $\nabla \mu$ is the gradient of chemical potential. To obtain this last quantity, Hull and Rimmer assumed that the chemical potential is $-\sigma_{a} \Omega$ midway between cavities and $-2 \gamma_{s} \Omega / r$ at the periphery of a cavity, where $\sigma_{\mathrm{a}}$ is the applied stress, $\gamma_{\mathrm{s}}$ is the surface energy, and $r$ is the radius of the cavity. By assuming a linear variation in chemical potential between these two points, they obtained the following expression for the flux:

$$
J=\left(D_{\mathrm{b}} / \mathrm{kT}\right) \quad\left\{\frac{\sigma_{\mathrm{a}}-2 \gamma_{\mathrm{s}} / r}{l / 2}\right\} \simeq 2 D_{\mathrm{b}} \sigma_{\mathrm{a}} / \mathrm{kT} l
$$

where $l$ is the distance between voids and $2 \gamma_{\mathrm{s}} / r$ is assumed to be much less than the applied stress. The area through which this flux occurs is $2 \pi r \delta_{\mathrm{b}}$, where $\delta_{\mathrm{b}}$ is the effective grain boundary thickness. Since each atom that leaves the cavity increases the cavity's volume by $\Omega$, the total flux results in a net volume flow of matter,

$$
\mathrm{d} V / \mathrm{d} t=4 \pi r D_{\mathrm{b}} \delta_{\mathrm{b}} \sigma_{\mathrm{a}} \Omega / \mathrm{kT} l
$$

away from the cavity and onto the grain boundary.

If the cavities are spherical, this volume flow of matter results in a size rate of cavity growth of

$$
\mathrm{d} r / \mathrm{d} t=D_{\mathrm{b}} \delta_{\mathrm{b}} \sigma_{\mathrm{a}} \Omega / \mathrm{kT} l r
$$

Integrating this result, the time development of the cavity size is given by

$$
\begin{aligned}
r & =\left[\left(2 D_{\mathrm{b}} \delta_{\mathrm{b}} / l\right)\left(\sigma_{\mathrm{a}} \Omega / \mathrm{kT}\right) t+r_{\mathrm{i}}^{2}\right]^{1 / 2} \\
& \simeq\left[\left(2 D_{\mathrm{b}} \delta_{\mathrm{b}} / l\right)\left(\sigma_{\mathrm{a}} \Omega / \mathrm{kT}\right)\right]^{1 / 2} t^{1 / 2}
\end{aligned}
$$

where $r_{\mathrm{i}}$ is the initial cavity radius, which here is assumed to be much smaller than the current cavity size, $r$, at time $t$. Hull and Rimmer bypassed this result, eq (5), and calculated directly the time-to-fracture, $t_{\mathrm{f}}$, as the time for the cavities to grow to one-half their separation, $l / 2$, where they coalesce and failure ensues. Thus, their final result was

$$
\begin{aligned}
t_{\mathrm{f}} & =\left(l^{3} / 8 D_{\mathrm{b}} \delta_{\mathrm{b}}\right)\left(\mathrm{kT} / \sigma_{\mathrm{a}} \Omega\right) \\
& =\beta^{3}\left(\rho^{-3 / 2} / 8 D_{\mathrm{b}} \delta_{\mathrm{b}}\right)\left(\sigma_{\mathrm{a}} \Omega / \mathrm{kT}\right)^{-1}
\end{aligned}
$$


where $\rho\left(=\beta^{2} / l^{2}\right)$ is the number of cavity nuclei per unit area of grain boundary and $\beta$ is a geometric factor related to the coordination number of nearestneighbor cavities (for example, $\beta=1$ for a square array and $\beta=(4 / 3)^{1 / 4}$ for an hexagonal array). Although the time-to-fracture and its stress dependence are the important parameters from an engineering perspective, the intermediate result of cavity size as a function of time, and also of cavity size distributions, are the important quantities when making a nondestructive assessment of creep damage for remaining lifetime.

Speight and Harris [11] objected to the assumption of a linear variation in chemical potential which is a direct consequence of performing the above, onedimensional calculation. Since the cavitation occurs in one plane, a two-dimensional calculation is required and this does not lead to a linear variation. Actually, Hull and Rimmer [10] realized this and had carried out an elegant calculation for the time-to-fracture in the appendix of their pioneering paper. In that appendix, they assumed a two-dimensional, square array of equal-sized, spherical nuclei. The solution for this geometry did not result in a linear gradient of chemical potential. Despite this modification, the calculated time-to-failure differed from the above equation, eq (6), only by a constant factor.

A further modification to the above model was suggested by Vitovek [12] who pointed out that as the cavities cover the boundaries, the stress on the remaining ligaments goes up. Then the assumption that the chemical potential is determined at all times by the applied stress must be dropped in favor of a stress that increases as the internal, loadbearing area decreases. Harris et al. [13] make this adjustment as well as that suggested by Speight and Harris [11] earlier, and find that the Hull and Rimmer model predicts $^{2}$

\footnotetext{
${ }^{2}$ In deriving the equation which ultimately leads to their form of eq (7). Speight and Harris [11] assumed that the stress midway between cavities is given by the remote stress, or by an adjusted ligament stress [13]. This is a valid approximation for small cavities; but as their results show, the stress can vary substantially across the grain boundary ligament for larger cavity sizes. Accordingly, in this regime the remote stress should be equated to the average stress on the grain-boundary ligament. This modification was first suggested by Raj and Ashby [14] and their model equates the remote stress to the average stress in the circular catchment area surrounding each cavity. This idea should be extended over the entire grain boundary area which includes the area excluded by the close packing of circles. We have made this modification in writing both eqs (7) and (9).
}

$$
\begin{aligned}
t_{\mathrm{f}} & =t_{\mathrm{HR}}\left(15 \pi^{2}-140-\pi \beta^{2}\right) / 60 \\
& \simeq t_{\mathrm{HR}} / 13.6 \quad \text { (for a hexagonal array) }
\end{aligned}
$$

where $t_{\mathrm{HR}}$ is the combination of material parameters $\left(D_{\mathrm{b}}, \delta_{\mathrm{b}}, l, \Omega\right)$ and experimental variables $\left(\sigma_{\mathrm{a}}\right.$ and $\left.\mathrm{T}\right)$ that represent the time-to-failure in the simple HullRimmer calculation presented above [see eq (6)]

$$
t_{\mathrm{HR}} \equiv\left(l^{3} / 8 D_{\mathrm{b}} \delta_{\mathrm{b}}\right)\left(\mathrm{kT} / \sigma_{\mathrm{a}} \Omega\right)
$$

In addition to the integrated result of eq (7), Harris et al. [13] also give a cavity growth relationship, which for short times has the form

$$
t / t_{\mathrm{HR}}=(2 / 3) x^{3} \ln (1 / x)
$$

where $x$ is the normalized cavity size $(2 r / l)$. Although this relationship cannot be inverted analytically to give the time dependence of the cavity size, one can easily demonstrate that initial cavity growth rates and cavity sizes are faster for this modified Hull-Rimmer model than for the simple calculation, eq (5).

One fault of the Hull-Rimmer model, whether in its simple or modified form, is that the cavities are assumed to be spherical. Only very small cavities are ever observed to be spherical. Usually, they are ellipsoidal or lenticular in cross-section. As the cavities grow, their shape frequently becomes more eccentric and they sometimes resemble long, thin cracks. An ellipsoidal cavity consumes more grain boundary than a spherical cavity for the same volume flux. This means that the above model, which assumes spherical cavities, will generally overestimate the time-tofracture.

The reason for crack-like cavities is apparent. The shape of a growing cavity is determined by the interaction between volume or boundary diffusion (the growth mechanism) and surface, volume, or vapor diffusion (spheroidizing mechanisms). Surface diffusion is generally the dominant rounding influence, while grain boundary diffusion is the principal growth mechanism. As the cavities enlarge, spheroidizing mechanisms become less effective and the cavities become more eccentric or penny-shaped.

Chuang and Rice [15] consider a crack-like creep cavity advancing at a steady state velocity $v$ along the interface between two grains by the diffusive transport of atoms into the boundary ahead of the tip. They assume that spheroidization of the cavity is accomplished by surface diffusion, and that the cavity 
grows under steady-state conditions so that it retains a constant profile which is described by a crack-tip radius of curvature, $r_{\text {tip }}$, and a crack opening width, $2 w$, a few radii back from the crack tip. Solving the surface diffusion problem they find that the crack halfthickness is given by

$$
w \simeq 0.98 \sqrt{2-\gamma_{\mathrm{b}} / \gamma_{\mathrm{s}}}\left[D_{\mathrm{s}} \delta_{\mathrm{s}} \gamma_{\mathrm{s}} \Omega / \mathrm{kT} v\right]^{1 / 3}
$$

where $\gamma_{s}$ and $\gamma_{b}$ are the surface free energy and grainboundary free energy, respectively; $D_{\mathrm{s}}$ is the surface diffusivity; and $\delta_{\mathrm{s}}$ is the thickness of the surface diffusion layer (i.e., the surface density of diffusing atoms times the atomic volume). The radius of curvature adjacent to the crack tip is given by

$$
\begin{aligned}
r_{\text {tip }} & \simeq 0.93\left[D_{\mathrm{s}} \delta_{\mathrm{s}} \gamma_{\mathrm{s}} \Omega / \mathrm{kT} v\right]^{1 / 3} / \sqrt{2-\gamma_{\mathrm{b}} / \gamma_{\mathrm{s}}} \\
& \simeq 0.95 w /\left(2-\gamma_{\mathrm{b}} / \gamma_{\mathrm{s}}\right) .
\end{aligned}
$$

Since $\gamma_{\mathrm{b}}$ is usually about one-half $\gamma_{\mathrm{s}}$ (corresponding to a dihedral angle of about $76^{\circ}$ ), eq (11) becomes approximately

$$
\begin{aligned}
w & \simeq 1.57 r_{\text {tip }} \\
& \simeq 1.20\left[D_{\mathrm{s}} \delta_{\mathrm{s}} \gamma_{\mathrm{s}} \Omega / \mathrm{kT} v\right]^{1 / 3} .
\end{aligned}
$$

This shows that the faster a crack grows, the thinner it becomes. Alternatively, the crack velocity at a given temperature is inversely proportional to the third power of the crack thickness. Hence, the time-tofracture for thin cracks or cavities is much less than that for spherical cavities. Chuang et al. [16] extend this analysis to calculate the time-to-fracture when the life is determined by the growth of cavities whose shape is determined by the interaction of spheroidizing and growth mechanisms. They find that

$$
t_{\mathrm{f}} \simeq\left(\frac{\mathrm{kT} l \gamma_{\mathrm{s}}^{2}}{35 D_{\mathrm{s}} \delta_{\mathrm{s}} \Omega \sigma_{\mathrm{a}}^{3}}\right)\left(2-\gamma_{\mathrm{b}} / \gamma_{\mathrm{s}}\right)^{3 / 2}\left(\frac{\bar{F}}{\sqrt{(1+\bar{F})-1}}\right)^{3}
$$

where

$\bar{F}=\frac{4 \sigma_{\mathrm{a}} l\left(D_{\mathrm{s}} \delta_{\mathrm{s}} / D_{\mathrm{b}} \delta_{\mathrm{b}}\right)}{3 \gamma_{\mathrm{s}} \sqrt{2-\gamma_{\mathrm{b}} / \gamma_{\mathrm{s}}}} \bar{q}$

and $\bar{q} \simeq 0.6$ is the average value of a slowly varying function of the cavity size [16]. Equation (13) has two limiting forms which depend on the relationship between a combination of material properties and the applied stress level. At one extreme, failure time is independent of grain-boundary diffusion and varies as the inverse third power of stress

$$
t_{\mathrm{f}} \simeq\left(\frac{8 \mathrm{kT} l \gamma_{\mathrm{s}}^{2}}{35 D_{\mathrm{s}} \delta_{\mathrm{s}} \Omega \sigma_{\mathrm{a}}^{3}}\right)\left(2-\gamma_{\mathrm{b}} / \gamma_{\mathrm{s}}\right)^{3 / 2}
$$

when $\sigma_{\mathrm{a}}<0.8\left(\gamma_{\mathrm{s}} / l\right)\left(D_{\mathrm{b}} \delta_{\mathrm{b}} / D_{\mathrm{s}} \delta_{\mathrm{s}}\right)$; whereas at the other limit, failure time is controlled by both surface and grain-boundary diffusion and varies as the inverse $3 / 2$ power of stress

$$
t_{\mathrm{f}} \simeq\left(\frac{\mathrm{kT}}{35 D_{\mathrm{s}} \delta_{\mathrm{s}} \Omega}\right)\left[\left(\frac{64 \bar{q}^{3}}{27}\right)\left(\frac{l^{5} \gamma_{\mathrm{s}}}{\sigma_{\mathrm{a}}^{3}}\right)\left(\frac{D_{\mathrm{s}} \delta_{\mathrm{s}}}{D_{\mathrm{b}} \delta_{\mathrm{b}}}\right)^{3}\right]^{1 / 2}
$$

when $\sigma_{\mathrm{a}}>150\left(\frac{\gamma_{\mathrm{s}}}{l}\right)\left(\frac{D_{\mathrm{b}} \delta_{\mathrm{b}}}{D_{\mathrm{s}} \delta_{\mathrm{s}}}\right)$

This concern over the stress dependence of the time-to-fracture arises from a discrepancy between the theoretical predictions and experimental fact. The Hull and Rimmer model [10], and Speight and Harris modifications [11,13], predict that the time-to-fracture should vary inversely with the first power of stress. Indeed, experiments by Raj [17] have indicated that this may be the case in bicrystal specimens. However, as shown above, the Chuang et al. model [16], which considers cavity shape, predicts that under certain conditions the fracture lifetime will be inversely proportional to a power of applied stress between $3 / 2$ and 3 . Thus, cavity shape can play an important part in determining how quickly a component will fracture by grain-boundary cavitation. By pre-nucleating cavities in silver and other metals, Goods and Nix [18] found times-to-failure that varied inversely with approximately the third power of stress, thus providing extremely strong experimental support for the theory of Chuang et al. [16].

Despite this support for the Chuang-Rice model $[15,16]$ by creep lifetime measurements on precavitated metals, the situation is not as clear-cut for the case where cavities are nucleated naturally on the grain boundaries. Experimental creep fracture work generally indicates a stronger inverse power-law stress dependence than given by either the Hull-Rimmer model or the Chuang-Rice model. To illustrate this dependence, creep fracture results for various metals and alloys [19-22] have been analyzed and are 
presented in table 1 . The stress exponent, $n$, given in this table is defined by the equation

$$
t_{\mathrm{f}}=B \sigma_{\mathrm{a}}^{-n}
$$

where $B$ is a constant of proportionality. The weakest stress dependences occur for the longest tests or at the highest temperatures, where $n$ is found to be about 3 or 4; but for shorter times-to-fracture, $n$ becomes quite large.

Table 1. Stress dependence of time-to-fracture for selected metals.

\begin{tabular}{lccrl}
\hline \multicolumn{1}{c}{ Metal } & $T / T_{\mathrm{M}}{ }^{1}$ & \multicolumn{1}{c}{$t_{\mathrm{f}}$} & $n$ & \multicolumn{1}{c}{ Reference } \\
\hline 316 S.S. & 0.54 & $4-20 \mathrm{yr}$ & 3.6 & Simmons and \\
& 0.60 & $2 \mathrm{w}-6 \mathrm{mo}$ & 3.8 & van Echo [5] \\
& 0.54 & $1 \mathrm{~d}-6 \mathrm{mo}$ & 6.3 & \\
& 0.60 & $1 \mathrm{~d}-2 \mathrm{w}$ & 5.3 & \\
& 0.54 & $\frac{1}{2} \mathrm{~h}-3 \mathrm{~h}$ & 14.3 & \\
304 S.S. & 0.60 & $\frac{1}{4} \mathrm{~h}-\frac{3}{4} \mathrm{~h}$ & 12.5 & \\
& 0.52 & $1-3 \mathrm{yr}$ & 3.8 & Simmons and \\
1Cr $\frac{1}{2}$ Mo Steel & 0.45 & $1-10 \mathrm{yr}$ & 3.7 & Bennewitz [19] \\
Lead & 0.50 & $1-5 \mathrm{yr}$ & 3.0 & Gohn et al. [20] \\
Tungsten & 0.83 & $1-10 \mathrm{~d}$ & 4.4 & Conway et al. [21] \\
Iron & 0.48 & $10 \mathrm{~h}-1 \mathrm{w}$ & 10.1 & Fields et al. [1] \\
& 0.54 & $3 \mathrm{~h}-1 \mathrm{w}$ & 9.0 & \\
Copper & 0.31 & $3-9 \mathrm{w}$ & 13.5 & Carreker and \\
& 0.52 & $2 \mathrm{~d}-1 \mathrm{w}$ & 4.1 & Hibbard [22] \\
\hline
\end{tabular}

\footnotetext{
${ }^{1} T / T_{\mathrm{M}}$ is the homologous temperature, where $T_{\mathrm{M}}$ is the melting temperature; and the stress exponent $n$ is defined by $t_{\mathrm{f}} \propto \sigma^{-n}$.
}

Although, as discussed above, the predominance of experimental evidence is related to the power of the stress dependence, there are at least two other important distinctions between the two models that are amenable to experimental investigation. The first is the density of cavities on the grain boundary, as defined, for example, by $\rho\left(\propto 1 / l^{2}\right)$. In the Hull-Rimmer model, both the time-to-failure and the time required to grow to a given cavity size are proportional to $\rho^{-3 / 2}$ (see eqs (6) and (9), respectively); whereas in the Chuang-Rice model, this dependence varies from $\rho^{-1 / 2}$ to $\rho^{-5 / 4}$ depending upon the applied stress and the material properties as defined by the two limiting cases (see eq (15)). The other distinction between the two models is the cavity growth rate. The HullRimmer model predicts a complex time dependence as prescribed by eq (9); whereas for small cavities, the Chuang-Rice model predicts a constant cavity growth rate or a linear time dependence of the normalized cavity size ${ }^{3}$,

$$
(2 r / l) \simeq 16 t / 35 t_{\mathrm{CR}}
$$

where $t_{\mathrm{CR}}$ is the time-to-failure as given by either eq (13) or one of its limiting forms, eq (15).

Based on these differences, alternative experimental techniques, such as small angle neutron scattering measurements, might help to elucidate the nature of cavity nucleation kinetics and cavity growth rates by providing information about cavity size distributions and how they evolve with time.

Returning, however, to the concern over the high powers of the stress dependence, Dyson [23], Rice and Needleman [24], and Rice [25] introduced the idea of constrained cavity growth to explain this. At high growth rates, the atoms diffusing away from the cavities cannot be distributed uniformly along the grain boundary. This effect locally unloads the regions around the cavities. Growth then requires the time dependent plastic straining of the matrix (i.e., creep) to counteract this load-shedding. Using this idea, the constrained cavity growth model predicts the Monkman-Grant [26] relation:

$$
t_{\mathrm{f}} \dot{\epsilon}_{\text {ss }}=\text { constant }
$$

where $\dot{\epsilon}_{\mathrm{ss}}$ is the steady state creep rate. This relation is a commonly found experimental result. The high powers listed in table 1 might then be explained by this constrained cavity growth model.

The above models all assume that all nucleation occurs at the onset of loading and that all the nuclei begin to grow at the start of the test. Greenwood [27] shows that most experimental data are consistent with a model in which the number of cavities is a linear function of strain, i.e., nucleation occurs throughout the life of a specimen. Indeed, the fact that Goods and Nix [18] had to prenucleate cavities to obtain the Chuang result indicates that nucleation is an important step in the creep fracture of some metals. This is another area where a technique such as small angle neutron scattering can be expected to provide vital information regarding this creep failure mechanism.

To include continuous nucleation in their analyses, Raj and Ashby [14] consider quantitatively two

\footnotetext{
${ }^{3}$ This equation is derived in the same spirit as Chuang et al. [16] calculated the time-to-failure.
} 
models for nucleating new cavities throughout a test: one without grain boundary sliding, and one with grain boundary sliding. Without grain boundary sliding, subcritical or embryonic cavities can become stable and grow by being thermally activated over the nucleation barrier. In this case, they applied classical nucleation theory. The change in free energy on forming a cavity is calculated by balancing the energy released when the applied forces do work against the energy absorbed by the creation of new interfacial area:

$$
\Delta G=-r^{3} F_{\mathrm{v}}(\psi) \sigma_{\mathrm{a}}+r^{2}\left[\gamma_{\mathrm{s}} F_{\mathrm{s}}(\psi)-\gamma_{\mathrm{b}} F_{\mathrm{b}}(\psi)\right]
$$

where $F_{\mathrm{s}}$ and $F_{\mathrm{b}}$ are the functions that relate the surface area of the cavity and the grain-boundary area, respectively, to the cavity radius squared; $F_{\mathrm{v}}$ is a similar function for the cavity volume; and $\psi$ is the cavity dihedral angle given by $\arccos \left(\gamma_{\mathrm{b}} / 2 \gamma_{\mathrm{s}}\right)$. Using the definitions of the functions $F_{\mathrm{v}}, F_{\mathrm{s}}$, and $F_{\mathrm{b}}$, eq (19) has been shown to have a maximum at a critical radius [14]

$$
r_{\mathrm{c}}=2 \gamma_{\mathrm{s}} / \sigma_{\mathrm{a}}
$$

below which cavities tend to shrink and above which they tend to grow. Substituting this critical radius into eq (19), gives the activation energy for nucleation:

$$
\Delta G_{\mathrm{c}}=r_{\mathrm{c}}^{3} F_{\mathrm{v}}(\psi) \sigma_{\mathrm{a}} / 2=4 \gamma_{\mathrm{s}}^{3} F_{\mathrm{v}}(\psi) / \sigma_{\mathrm{a}}^{2}
$$

The area density of critical nuclei, i.e., those that prefer to neither shrink nor grow, on the grain boundary is

$$
\rho_{\mathrm{c}}=\rho_{\max } \exp \left[-\Delta G_{\mathrm{c}} / \mathrm{kT}\right]
$$

where $\rho_{\max }$ is the maximum density of possible nucleation sites there. The nucleation rate can be obtained from $\rho_{c}$ by multiplying eq (22) by the timedependent probability, $P(\mathrm{t})$, of adding one vacancy to a critical nucleus. Raj and Ashby [14] derive $P(\mathrm{t})$ from the vacancy jump frequency and from the probability of finding a vacancy at the perimeter of a critical nucleus. They obtain

$$
P(\mathrm{t})=\left(4 \pi \gamma_{\mathrm{s}} / \sigma_{\mathrm{a}} \Omega\right)\left(D_{\mathrm{b}} \delta_{\mathrm{b}} / \Omega^{1 / 3}\right) \exp \left[\sigma_{\mathrm{a}} \Omega / \mathrm{kT}\right]
$$

By combining eqs (21), (22), and (23), the nucleation rate is found to be

$$
\begin{aligned}
\dot{\rho}= & \left(4 \pi \gamma_{\mathrm{s}} D_{\mathrm{b}} \delta_{\mathrm{b}} / \sigma_{\mathrm{a}} \Omega^{4 / 3}\right)\left(\rho_{\max }-\rho\right) \\
& \times \exp \left[\left(\sigma_{\mathrm{a}} \Omega-4 \gamma_{\mathrm{s}}^{3} F_{\mathrm{v}}(\psi) / \sigma_{\mathrm{a}}^{2}\right) / \mathrm{kT}\right]
\end{aligned}
$$

The dominant term in the above equation is $\exp \left(-1 / \sigma_{\mathrm{a}}^{2}\right)$. How does this term in the nucleation rate affect the time-to-fracture? To obtain the time-tofracture, it is necessary to perform a double integration over time because nucleation occurs simultaneously with cavity growth. Since exponential arguments are unaffected by integration, the strong stress dependence of nucleation will be carried into the time-to-fracture. Such an exponential dependence on stress can mimic any power-law dependence of the form

$$
t_{\mathrm{f}}=B \quad \sigma_{\mathrm{a}}^{-n} \quad(\text { see eq }(16)),
$$

regardless of the value of $n$, over certain ranges of stress.

The second type of nucleation mechanism examined by Raj and Ashby [14] is based on grain boundary sliding. If there is an inclusion on a sliding grain boundary, then this sliding is either accommodated by flow of atoms around the particle or else a gap opens up. Accommodation is possible at low stresses leading to low sliding rates, but as the stress and sliding rate increase the flow intensification at the inclusion also increases until cavity nucleation occurs. Thus, the nucleation rate and the number of nuclei are dependent on strain and strain rate. Since the rate of fracture is proportional to the rate of nucleation, an additional stress dependence similar to that for the strain rate will enter the expression for the time-tofracture. In this way, nucleation considerations can be used to predict the Monkman-Grant relation.

\section{Experimental Techniques to Study Creep Cavitation}

We have discussed four theories: Hull and Rimmer [10], coupled grain boundary and surface diffusion controlled growth [15], constrained cavity growth [23], and continuous cavity nucleation [14]. Of the numerous theoretical works on creep cavitation, the models discussed above are the ones supported by experiment. To some extent, the above models are mutually exclusive. While it is likely that under different experimental circumstances, different models are appropriate, the situation is confusing. Most often, support for one model over another is determined 
from the stress dependence of the time-to-fracture. Considerably more persuasive support would come from direct measures of cavity nucleation rates and subsequent cavity growth rates.

Attempts to experimentally measure these two quantities have not been conclusive. The two most common techniques are metallography and density. Metallographic techniques include microscopy techniques ranging from optical measurements on polished surfaces to transmission electron microscopy (TEM) measurements on thin foils. The optical techniques suffer from a lack of resolution and the difficulties inherent in preparing a surface without grossly altering the cavities. The TEM studies usually have had difficulty finding any cavities due to the limited material volume which is sampled in each TEM specimen. When a cavity is found, it is not clear that it is representative of typical cavities in the material. Another metallographic technique is to fracture a specimen along its grain boundaries at low temperature after it has been crept at elevated temperature, and to measure the cavitation in a scanning electron microscope (SEM). This is the most successful of the metallographic techniques. It is always a concern, however, that the low temperature fracture surface has selected the most heavily cavitated boundaries and may not be representative of the overall cavitation.

High precision density measurements usually employ Archimedes' principle. Since the buoyant fluid will probably penetrate surface cracks, this technique will be mainly sensitive to bulk cavitation. This technique can only tell the total volume of cavities. For this reason, it cannot be used to study continuous nucleation, which requires a knowledge of cavity size distributions.

Two new techniques for studying the cavitation phenomenon are small angle neutron scattering (SANS) and $\mathrm{x}$-ray topography or $\mathrm{x}$-radiography. SANS work has already been performed at NBS and other laboratories. These initial studies have demonstrated that SANS is very sensitive to cavitation and may be the most powerful technique yet tried for studying nucleation of creep cavities. X-ray techniques have not been tried yet, but they may provide information concerning the shape and growth rate of creep cavities measured in-situ.

SANS is performed by measuring the angular dispersion of an initially collimated beam of monochromatic neutrons. This dispersion can be related to the size and size distribution of scattering particles, or cavities, by using elements of established diffraction theory. In addition to the present studies,
SANS studies have been carried out on creepdamaged stainless steel [28], superalloys [29], and copper [30]. In every case, the scattering was observed to increase as the damage increased. The interpretation of the results for the stainless steel and the superalloys was clouded by changes in the precipitate size distribution that occurred during the test. Furthermore, the scattering in the stainless steel was attributed to cavities although other techniques, such as TEM, never revealed the presence of creep cavities. SANS studies of creep cavitation at NBS to date have centered on 304 stainless steel. As will be discussed below, the use of unstressed reference specimens and a carbide stabilizing heat treatment have virtually eliminated the ambiguities arising from precipitate redistribution during the test. Also, microscopic studies have revealed intergranular cavities in specimens that have been tested long enough to grow the cavities to resolvable sizes. Therefore, it is valid to attribute the neutron scattering to cavitation.

\section{SANS Study of Cavitation in 304 Stainless Steel}

304 stainless steel used in high temperature applications consists mainly of austenite grains and grain-boundary metal carbides. The carbide-austenite interface at the grain boundary is a preferential site for cavity nucleation, which at high temperatures can be readily activated with low applied stresses by creep deformation and/or grain-boundary sliding. The resulting cavities then grow, link, and ultimately cause failure. The early stages of this process are extremely slow and the creep cavities, due to their small size, are not easily detectable or quantifiable. It is these early stages of nucleation and cavity growth that are examined here through the use of small angle neutron scattering.

The structure after cavitation consists of grainboundary cavities and two material phases (austenite and carbides). The unequivocal determination of cavity size, or size distribution, from a SANS spectra requires that the creep-induced changes in the carbide volume fraction, size distribution, and spacial distribution do not contribute significantly to the scattered intensity. By adopting special experimental procedures, the effects due to the carbides could be minimized. The following two procedures were employed:

1. All samples were given a stabilizing heat treatment of $40 \mathrm{~h}$ at $775^{\circ} \mathrm{C}$ prior to creep 
testing. This resulted in a very coarse and stable carbide distribution.

2. A heat-treated, but unstressed, reference sample was placed in the furnace with each creep specimen. In each case, the SANS spectra of the unstressed sample was subtracted from that of the stressed specimen to obtain the SANS spectra of the creep damage.

The SANS studies were carried out at the NBS reactor facility using a converging collimation and a neutron wavelength of $0.625 \mathrm{~nm}$. With this choice of collimation and wavelength parameters, a scattering vector $(\mathbf{Q})$ range of 0.05 to $1.5 \mathrm{~nm}^{-1}\left(0.005\right.$ to $\left.0.15 \AA^{-1}\right)$ could be measured in these experiments. This $\mathbf{Q}$ range measures the scattering contribution of the inhomogeneities in the size range of 4 to $120 \mathrm{~nm}$. Detailed theory and procedures for analyzing SANS data have been described by Kortorz [31]. A few relevant features will be discussed here. If the scattered intensity is denoted by $I$, the differential scattering cross-section is given by

$$
d \Sigma / d \Omega=c I / M T d
$$

where $d$ is the sample thickness, $M$ is the total monitor count (or scattering time), $T$ is the sample transmission, and $c$ is a combination of experimental constants related to the SANS facility. Creep damage is related to the difference in differential scattering cross-sections between the crept sample (C) and the reference sample $(R)$ :

$$
\left[\frac{d \Sigma_{\mathrm{C}}}{d \Omega}-\frac{d \Sigma_{\mathrm{R}}}{d \Omega}\right]=c\left[I_{\mathrm{C}} / M_{\mathrm{C}} T_{\mathrm{C}} d_{\mathrm{C}}-I_{\mathrm{R}} / M_{\mathrm{R}} T_{\mathrm{R}} d_{\mathrm{R}}\right]
$$

Accordingly, all intensities in the following discussion have been normalized by sample thickness, transmission, and scattering time. We now assume that the entire difference in scattering is due to creep cavities alone. This is justified by the use of the special procedures described above. Figure 4 shows a scattering pattern for one set of creep and reference samples. This figure shows, as did all the samples, that the crept sample scatters more strongly than the reference sample. This result supports the assumption that cavities are the predominant scattering feature, because carbide coarsening would show up as decreased scattering. The data are replotted in figure 5 as the difference spectra.

One parameter of major interest is the growth of average cavity size as a function of time under load. A

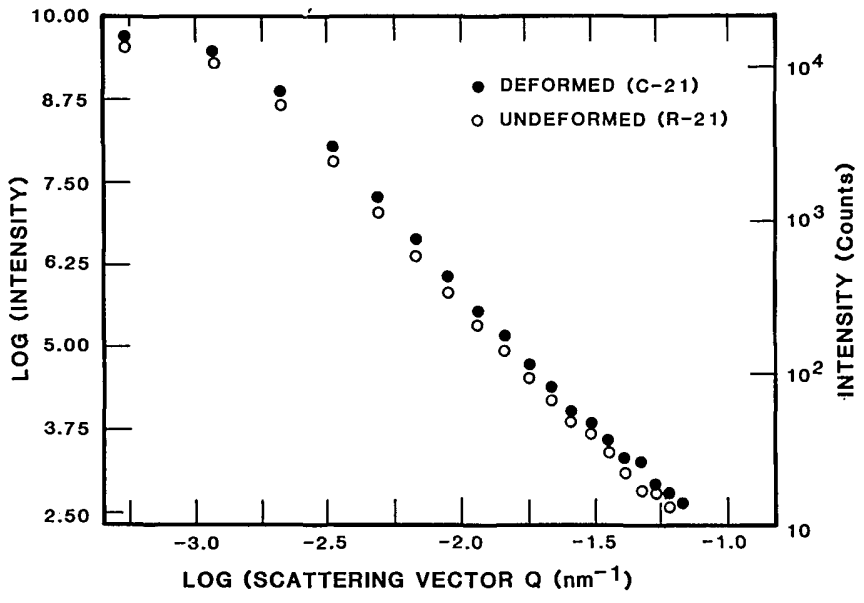

Figure 4-SANS spectra for a 304 stainless steel specimen which was deformed under a $145 \mathrm{MPa}$ tensile stress at $600^{\circ} \mathrm{C}$ for $1493 \mathrm{~h}$ (specimen C-21) and for an undeformed reference specimen (specimen R-21).

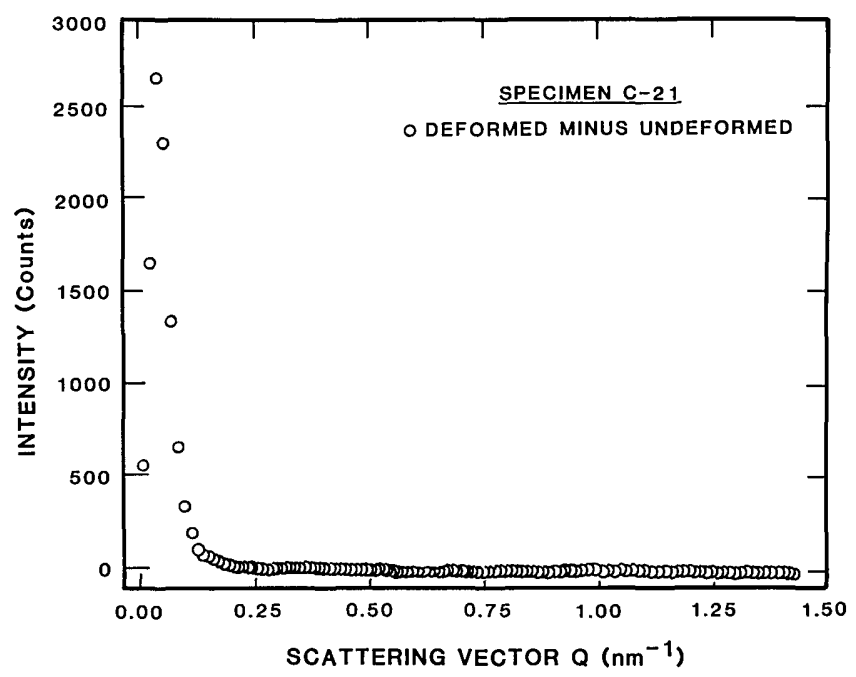

Figure 5-SANS difference spectra from figure 4 showing the scattering due to creep-induced cavities.

Guinier analysis [31] was performed on spectra like figures 4 and 5 to obtain an average cavity size. Guinier showed that for a randomly distributed monosized, spherical heterogeneity, the intensity (I) and scattering vector $(\mathbf{Q})$ relationship can be described by the equation

$$
I=I_{0} \exp \left[-R_{\mathrm{g}}^{2} \mathbf{Q}^{2} / 3\right]
$$

Here $R_{\mathrm{g}}$ is the radius of gyration of the cavities and for spherical cavities it is related to the cavity radius $(R)$ by the equation

$$
R=\sqrt{5 / 3} R_{\mathrm{g}} .
$$


If the cavities are not of one size, then the utility of the Guinier approximation is limited to a very small $\mathbf{Q}$ range near zero. Figure 6 is a Guinier plot $(\log I$ versus $\mathbf{Q}^{2}$ ) for the difference spectra of figure 5 giving information about the creep cavitation damage. It is clear from this plot that cavities in this material must have a nonuniform size distribution, as a straight line in these plots can be fitted only over a narrow $\mathbf{Q}$ region near the peak. The cavity diameter obtained by this procedure normally overestimates the contribution of large cavities, yet it is a very useful parameter to study cavity growth as a function of creep time while degradation is in progress. Using a least square fit subroutine, the above equation for $I$ was fit to the

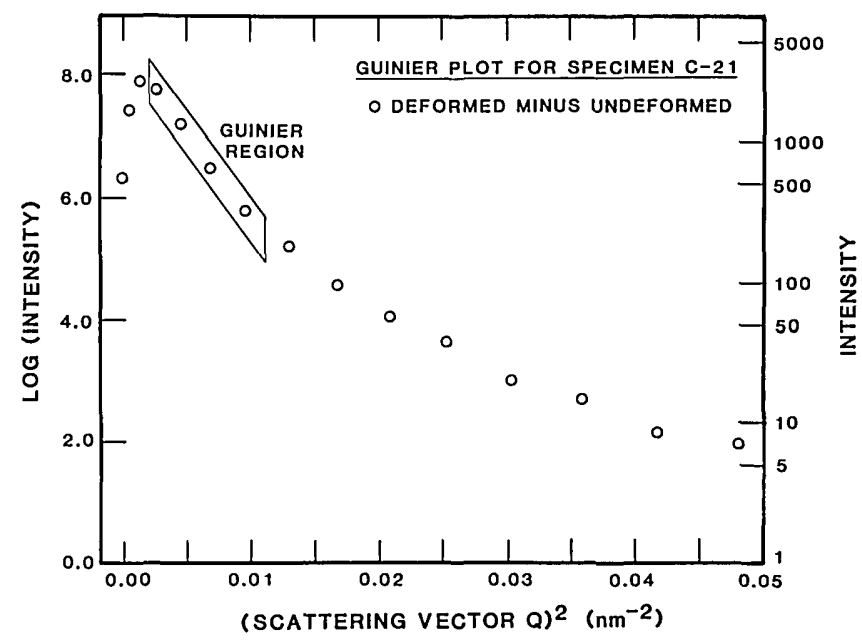

Figure 6-Plot of the Guinier region for the difference spectrum of figure 5 showing the creep damage in specimen C-21.

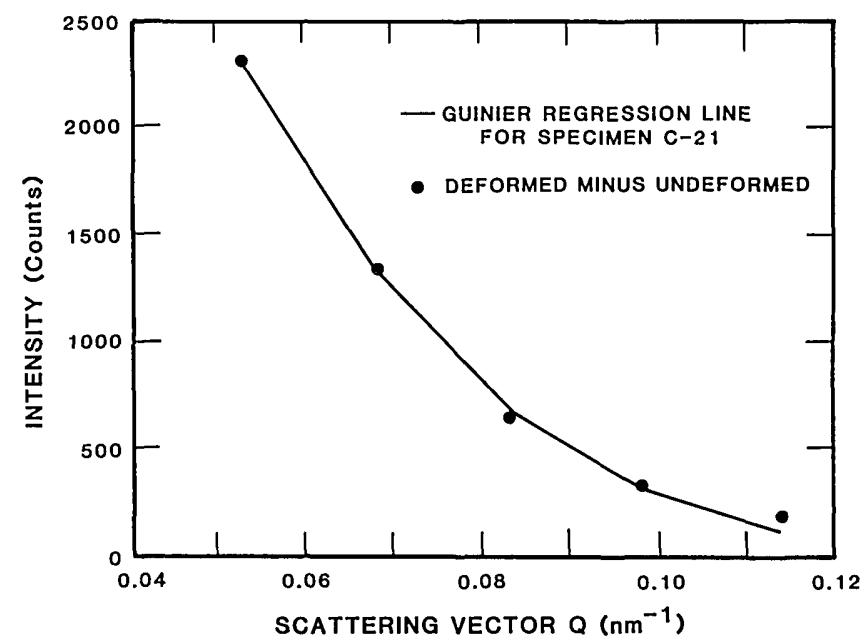

Figure 7-Least squares regression analysis of the linear Guinier region in figure 6 and the fitted, appropriate data points.
Table 2. Growth of creep cavities as a function of time at constant stress in 304 stainless steel.

\begin{tabular}{|c|c|c|c|}
\hline \multirow[t]{2}{*}{ Sample } & \multicolumn{2}{|c|}{ Creep parameters } & \multirow{2}{*}{$\begin{array}{l}\text { Average cavity } \\
\text { diameter-Guinier } \\
\text { approximation }(\mathrm{nm})\end{array}$} \\
\hline & Applied stress & Time (h) & \\
\hline \multirow[t]{2}{*}{ C-21 } & $\begin{array}{l}145 \mathrm{MPa} \\
(21 \mathrm{ksi})\end{array}$ & 745 & 34.6 \\
\hline & & 1493 & 77.0 \\
\hline \multirow[t]{2}{*}{ C-18 } & $\begin{array}{l}124 \mathrm{MPa} \\
(18 \mathrm{ksi})\end{array}$ & 789 & 26.6 \\
\hline & & 1537 & 76.3 \\
\hline
\end{tabular}

data points in the relevant $\mathbf{Q}$ range. A set of experimental data points and the regression lines are shown in figure 7. (Note that this figure is plotted as $I$ versus $\mathbf{Q}$, so that the regression line is not straight.) $\mathbf{A}$ reasonably accurate fit was obtained in the selected $\mathbf{Q}$ range for this specimen as well as for the other creep specimens. The average cavity sizes derived for the various stresses and times under load at $600{ }^{\circ} \mathrm{C}$ are shown in table 2.

Table 2 shows that doubling the creep time at the same stress apparently doubles the average cavity size, apparently in agreement with the Chuang-Rice model, which predicts a constant cavity growth rate. Figure 8 shows cavity size as a function of creep time. If we include a point at the origin, the linear time dependence in the growth rate of the cavities is again apparent, but in the absence of more data points, only a limited significance can be attached to the trend in figure 8. Specimens have been tested to intermediate times and their SANS spectra will be determined shortly.

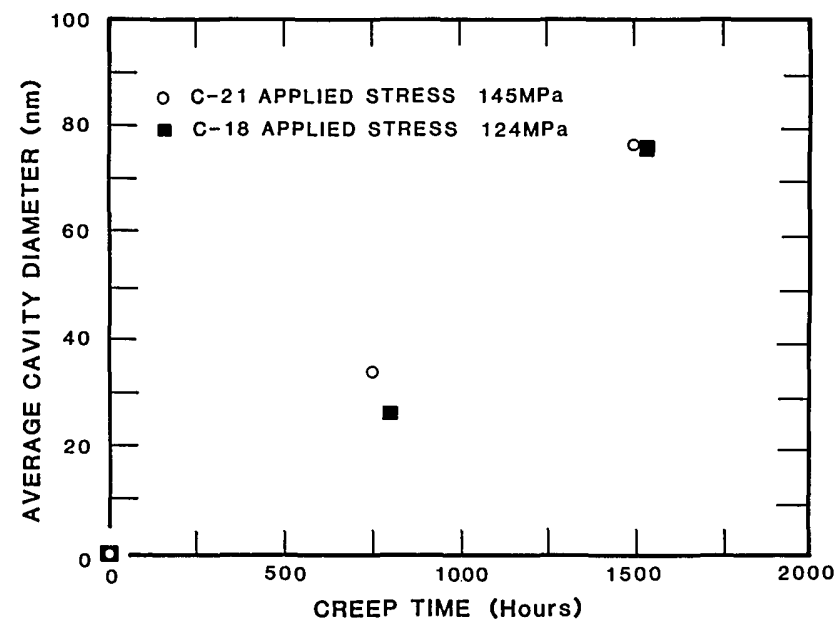

Figure 8-Average cavity diameter for 304 stainless steel specimens at $600{ }^{\circ} \mathrm{C}$ as a function of time and stress. 
From table 2, we see that specimen C-21, which was stressed at $145 \mathrm{MPa}(21 \mathrm{ksi})$, has a higher average cavity size after $750 \mathrm{~h}$ than specimen $\mathrm{C}-18$ which was stressed at $124 \mathrm{MPa}(18 \mathrm{ksi})$. After $1500 \mathrm{~h}$, we find that the average cavity size at these two stress levels is not too different. This could indicate that for longer creep times the size growth of cavities is less sensitive to small changes in applied stress. A more likely possibility, as noted by Weertman [30], is that when a sizable fraction of cavities grows large enough to scatter at $\mathbf{Q}$ values below the minimum resolvable $\mathbf{Q}$ in the experiments, the average size and volume fraction deduced from the standard SANS analysis are highly underestimated. Recent efforts have been made to extend SANS measurements to larger size ranges [32], for which other techniques have enough resolution to be effective. In this overlapping size range, SANS and these other techniques can be compared with each other to provide some assessment of their validity in the nonoverlapping size ranges.

The most important development demonstrated here is that we are capable of measuring cavities at early times when they are only 20 to $30 \mathrm{~nm}$ in diameter. This must be close to the nucleation event. No other technique, that we know of, is capable of resolving this initiation phenomenon. Further work should provide direct experimental information that will test the assumptions of the models discussed previously. Since these models are to be used for predictive purposes, they must be verified. SANS currently appears to be the best technique to characterize creep damage in order to test these theories.

\section{Conclusion}

Numerous theories of creep cavitation have developed over the years. The evaluation and further refinement of these theories has been impeded by a lack of experimental research in this area. Small angle neutron scattering studies at NBS and elsewhere have shown that this technique may provide the necessary tool for studying nucleation and early growth of cavities. Such a development would provide the impetus to further progress in this field.

We wish to thank S. M. Wiederhorn and T. R. Shives for reviewing this paper and for their helpful suggestions.

\section{References}

[1] R. J. Fields, T. Weerasooriya, and M. F. Ashby, Met. Trans. 11A, 333 (1980).

[2] M. F. Ashby, C. Gandhi, and D. M. R. Taplin, Acta Met. 27, 699 (1979).

[3] G. V. Smith, E. J. Dulis, and E. G. Houston, Trans. ASME 42, 935 (1950).

[4] E. J. Dulis, G. V. Smith, and E. G. Houston, Trans. ASME 45, 42 (1953).

[5] W. J. Simmons and J. A. van Echo, ASTM Data Series Publication DS5-s1 (1965).

[6] B.S.C.C., British Steel Corporation, p. 11, ISO/TC17 /SC10/ETP-SG (Secretariat 104) 138- MT/QF/54/74 (1972-73).

[7] A. Nadai and M. J. Manjoine, J. Appl. Mech. 63, A.77 (1941).

[8] R. K. Varma and B. F. Dyson, Scripta Met. 16, 1279 (1982).

[9] T.-J. Chuang, Int. J. Fracture 23, 229 (1983).

[10] D. Hull and D. E. Rimmer, Phil. Mag. 4, 673 (1959).

[11] M. V. Speight and J. E. Harris, J. Metal Science 1, 83 (1967).

[12] F. H. Vitovek, J. Materials Science 7, 615 (1972).

[13] J. E. Harris, M. O. Tucker, and G. W. Greenwood, Metal Science 8, 311 (1974).

[14] R. Raj and M. F. Ashby, Acta Met. 23, 653 (1975).

[15] T.J. Chuang and J. R. Rice, Acta Met. 21, 1625 (1973).

[16] T.J. Chuang, K. I. Kagawa, J. R. Rice, and L. B. Sills, Acta Met. 27, 275 (1979).

[17] R. Raj, Acta Met. 26, 341 (1978).

[18] S. H. Goods and W. D. Nix, Acta Met. 26, 739 (1978).

[19] J. H. Bennewitz, Joint International Conf. on Creep (Inst. of Mech. Engineers, London, 1963).

[20] G. R. Gohn, S. M. Arnold, and G. M. Boulton, Proc. ASTM 46, 990 (1946).

[21] J. B. Conway and P. N. Flagella, Creep Rupture Data for the Refractory Metals to High Temperatures (Gordon and Breach Publishers, New York, 1971).

[22] R. P. Carreker, Jr. and W. R. Hibbard, Jr., Acta Met. 1, 654 (1953).

[23] B. Dyson, Metal Science, 349 (1976).

[24] A. Needleman and J. R. Rice, Acta Met. 28, 1315 (1980).

[25] J. R. Rice, Acta Met. 29, 675 (1981).

[26] F. C. Monkman and N. J. Grant, Proc. ASTM 56, 593 (1956).

[27] G. W. Greenwood, Phil. Mag. 19, 423 (1969).

[28] M. H. Yoo, J. C. Ogle, J. H. Schneibel, and R. W. Swindeman, in Proc. 6th International Conf. on Strength of Metals and Alloys, Melbourne, Australia, Aug. 16-20, 1982 (Pergamon Press).

[29] H. Walther and P. Pizzi, SANS for Nondestructive Testing in Research Techniques in Nondestructive Testing, Vol. IV, Edited by R. S. Sharpe (Academic Press, 1980).

[30] J. R. Weertman, M. Yang, and M. Roth, Grain Boundary Cavitation in Copper Subjected to High Temperature Creep, Annex to Annual Report, Institut Laue-Langevin, Grenoble, France, p. 263.

[31] G. Kostorz, Neutron Scattering in Materials Science, Chapter 5 in Treatise in Materials Science and Technology, Vol. 15, Edited by H. Herman (Academic Press, New York, 1979).

[32] K. Hardman-Rhyne, N. F. Berk and E. R. Fuller, Jr., J. Res. Natl. Bur. Stand. (U.S.). 89(1): 00-00; 1984 JanuaryFebruary. 